$10.1117 / 2.1200611 .0501$

\title{
Generating imagery for forecasting terror threats
}

Greg Schmidt, Jason Goffeney, Jason Dalton, and Ruth Willis

Maps indicating threat levels based on key feature proximity, and incorporating event location uncertainty, are useful for planning countermeasures in the global war on terror.

Intelligence analysts and military planners need predictions about likely terrorist targets in order to better plan the deployment of security forces and sensing equipment. We have addressed this need using Gaussian-based forecasting and uncertainty modeling. Our approach excels at indicating the highest threats expected for each point along a travel path and for a 'global war on terrorism' mission. It also excels at identifying the greatest-likelihood collection areas that would be used to observe a target.

Our methods are extensions of Donald Brown's work ${ }^{1}$ on geospatial analysis and asymmetric-threat forecasting in the urban environment. He showed how to extract distinct signatures from associations made between historical event information and contextual information sources such as geospatial and temporal political databases. We have augmented this to include uncertainty estimates associated with historical events and geospatial information layers. ${ }^{2}$

\section{Event Forecasting Spatial Preferences}

The notion of spatial preferences has been used to find potential crime $^{1}$ and threat ${ }^{3}$ 'hot spots.' The premise is that a terrorist or criminal is directed toward a certain location by a set of qualities, such as geospatial features, demographic and economic information, and recent political events. Focusing on geospatial information, we assume the intended target is associated with features a small distance from the event location. We assign the highest likelihoods to the distances between each key feature and the event, and taper them away from these distances. This behavior is modeled using a kernel function centered at each of these distances. For a Gaussian kernel applied to a discretized map, the probability density function $\rho$ for a given grid cell $g$ and uncertainty estimates $u$ is given by

$$
\rho(g, u)=c \prod_{i=1}^{I} \frac{1}{N} \sum_{n=1}^{N} K\left(D_{i g}-D_{i n}+u\left(\phi_{E}, \phi_{F}\right)\right),
$$

where

$$
K(\theta)=\frac{1}{\sqrt{2 \pi \sigma^{2}}} e^{-\theta^{2} / 2 \sigma^{2}} .
$$

$D_{i g}$ is the distance from feature $i$ to the grid cell, $D_{i n}$ is the distance from the feature to event location $n, c$ is a constant, $\phi_{E}$ and $\phi_{F}$ are the position uncertainty for event and features respectively, $I$ is the total number of features, and $N$ is the total number of events. Figure 1(a) shows a sample forecast image based on this approach, denoting threat level with colors ranging from blue for lowest threat, through red for highest threat. For the same set of features and events, Figure 1(b) shows a more manageable forecast-in terms of allocating security resourcesdetermined by aggregating feature layers prior to generating the likelihood values.

\section{Modeling Uncertainty}

One of the most important aspects of forecasting is having an estimate of the confidence in the supporting numerical values. In numerical weather prediction, there is always a value of confidence assigned with each forecast. For example, predicting an $80 \%$ chance of rain implies that numerical weather models given input parameter variations, predicted eight out of ten tries that it would rain.

Similarly, for our event forecasts, we have identified three key sources of uncertainty. These are: first, positional uncertainty associated with geospatial locations for geographic, demographic, economic, political-event, and historical-event data; second, error associated with feature reduction; and finally, methodological error associated with the event forecasting algorithms. Here, we will focus only on the positional error of historical event locations.

The historical event record of the data we used included the date, location, type of attack, organization claiming responsibility, a description of what happened, and confidence of the recorded data. The confidence values for the locations are rated

Continued on next page 

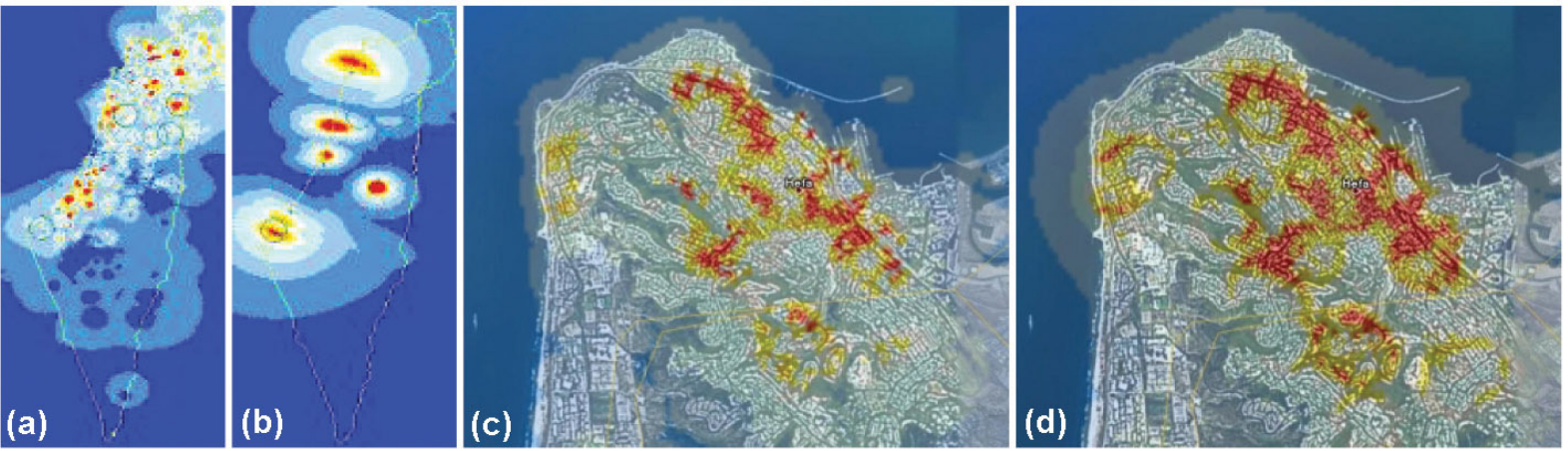

Figure 1. Examples of threat forecasts are shown, with threat likelihood coded by color, ranging from blue for lowest threat to red for highest threat. (a) Several high-level threats appear in this example. (b) Feature reduction of (a), achieved by aggregating geospatial information layers, provides a more manageable forecast for security resource allocation. (c) Map overlay of subregion hot spots. (d) Threat levels are more widely distributed when historical-event position uncertainties are incorporated in (c).

from 1 to 5 , with error values starting at $\pm 10 \mathrm{~m}$ and increasing by a power of 10 for each rank. The ratings represent analyst confidence in the precise event location. Error values in the event locations, $u_{E}$, are incorporated into the distance measurements by setting the feature-to-event distance, $D_{i n}$, to $D_{i n} \pm u_{E}$. We account for this variation by discretizing the distance range, and sampling by Monte Carlo simulation. Figures 1(c) and 1(d) show the impact of accounting for the uncertainty. These forecast images were converted to Google Keyhole Markup Language and are shown in the Google Earth program, ${ }^{4}$ overlaying the correct georegistered terrain.

\section{Conclusion}

We have explored approaches for generating forecast imagery used in future terror event predictions that include data uncertainty. Our future work will primarily focus on exploring the impact of more types of uncertainty.

\section{Author Information}

\section{Greg Schmidt, Jason Goffeney and Ruth Willis}

Advanced Information Technology

Naval Research Laboratory

Washington, D.C.

Greg Schmidt is a computer scientist at NRL, where he focuses on large-scale information, modeling uncertainty, and visualization topics, and received his PhD from Texas A\&M University. He has written a few papers on visualization topics for SPIE conferences.

Jason Goffeney is a computer scientist working for NRL as a contractor with ITT Corporation, and is working toward his PhD at University of Missouri-Columbia.
Ruth Willis is the head of the Advanced Information Technologies Branch at NRL, where she directs projects in geospatial analysis and reasoning. She received her PhD in industrial and organizational psychology at the University of South Florida.

\section{Jason Dalton}

Spatial Data Analytics (SPADAC)

Vienna, VA

Jason Dalton is the VP of Research and Development at SPADAC. He has ten years of experience in spatiotemporal forecasting, and is working toward his $\mathrm{PhD}$ at the University of Virginia.

\section{References}

1. D. Brown, J. Dalton, and H. Hoyle, Spatial forecast methods for terrorist events in urban environments, in Symposium on Intelligence and Security Informatics, Lecture Notes in Computer Science 3073, pp. 426-435, Springer-Verlag, 2004.

2. J. Goffeney, G. Schmidt, J. Dalton, J. D'Archangelo, and R. Willis, Forecast Visualizations for terrorist events, IEEE Visualization Conference, 2006.

3. H. Liu and D. Brown, Criminal incident prediction using a point-pattern-based density model, International Journal of Forecasting 19, pp. 603-622, 2003.

4. Google Earth: A 3D Interface to the Planet Last accessed October 19, 2006. http:/ / earth.google.com 